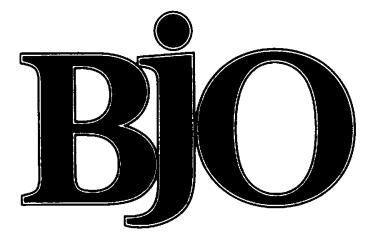

British Journal of Ophthalmology

\title{
Editorials
}

\section{Local resection of posterior uveal melanoma}

Although enucleation was once considered to be the only acceptable management for malignant melanoma of the posterior uvea (ciliary body and choroid), in recent years it has become controversial. ${ }^{1}$ This controversy over enucleation has contributed to the popularity of several other therapeutic methods. ${ }^{2}$ Depending on a number of clinical factors, current options to enucleation for uveal melanoma include a period of observation without interventional treatment, plaque radiotherapy, charged particle irradiation, trans-scleral lamellar resection, laser photocoagulation, and transpupillary thermotherapy. In this issue of the $B \mathcal{}$, Damato, Paul, and Foulds describe in two articles their further observations on trans-scleral resection of posterior uveal melanoma. Since this technique is difficult, it is only used in a few centres and it is not employed as commonly as other methods such as enucleation or plaque radiotherapy. Our group has used a technique of local resection of posterior uveal tumours that is quite similar to that of Damato and colleagues, and we agree that it is the preferred treatment in selected cases. ${ }^{34}$ We have now resected more than 250 tumours which involved the ciliary body and/or choroid and are generally impressed with the results.

Their first report ( $p$ 102) deals with risk factors for residual and recurrent tumour after trans-scleral local resection. It is not surprising that a melanoma which is located more posteriorly carries a greater chance of residual tumour after surgery because of the technical difficulty of removing tumours that are located very close to the optic disc and fovea. It is likewise not surprising that a larger tumour, located more posteriorly and of a more malignant cell type, has a greater chance of delayed local recurrence.

An interesting observation of these authors was that a lack of adjunctive plaque radiotherapy was associated with a greater chance of local tumour recurrence. Consequently, the authors suggest that supplemental plaque radiotherapy, laser photocoagulation, or cryotherapy should be employed routinely after local resection of uveal melanoma. This planned use of more than one modality reflects a new concept that is beginning to develop in the management of uveal melanoma. In the past, the ocular oncologist who managed a uveal melanoma would select a single method, such as enucleation, radiotherapy, local resection, or perhaps other treatments. Much has been written about the indications for each of these single modalities. However, little has been mentioned about planned combined therapeutic approaches. The planned use of local resection and plaque radiotherapy, as suggested by Damato and colleagues, is an example of such a combined therapeutic approach.

Our experience on the Oncology Service at Wills Eye Hospital also suggests that planned combined methods may be preferable in selected cases. It is becoming more common for us purposely to select a combination of these methods as part of the initial plan. For example, numerous patients have been treated with plaque radiotherapy followed by low energy, long exposure laser photocoagulation as advocated by Foulds and Damato. ${ }^{5}$ More frequently today, we employ plaque radiotherapy combined with transpupillary thermotherapy as recently proposed by Oosterhuis and associates. ${ }^{6}$ For tumours that are to be managed by local resection, we frequently plan to use plaque radiotherapy as supplemental treatment. Preenucleation radiotherapy is sometimes employed for patients who are to undergo enucleation for larger uveal tumours. In patients who are considered high risk for metastasis, enucleation combined with chemotherapy is currently being assessed. The observations of Damato and associates have supported our current belief that planned combined treatments may have a significant future role in the management of uveal melanoma.

The second paper by Damato and associates ( $p$ 109) describes the risk factors for metastatic uveal melanoma after trans-scleral local resection. As acknowledged by the authors, it is not surprising that advanced age, more malignant cell type, and greater tumour diameter were significantly associated with eventual metastasis. The association of superior location of the tumour with eventual metastasis is curious and difficult to explain. It is of interest that lack of adjunctive plaque radiotherapy was also associated with increased chance of metastasis. This again supports the argument for considering combined treatments in the initial therapeutic plan.

Since removal of posterior uveal tumours by local resection is a difficult and time consuming procedure which requires more complicated postoperative care, one may question the advisability of local resection in cases where there is little hope for any useful vision in the affected eye. Although in these latest papers the authors do not elaborate on their current indications for trans-scleral resection, the data that they present in these and earlier publications may provide insight as to when this procedure should be employed. This information would be of value to physicians who may employ local resection or who may wish to refer patients for that procedure. Based on their 
results and our personal experience, I would like to describe briefly what we believe to be the current relative indications and contraindications for local resection of ciliary body and choroidal melanoma. It is stressed that this represents a personal viewpoint which may differ somewhat from that of Damato and associates and other clinicians who may use local resection of posterior uveal melanoma.

The indications for local resection will necessarily vary from patient to patient and each case must be individualised depending on the entire clinical situation. However, in general, local resection should be considered when the patient is middle aged or younger, and when the tumour has a more anterior location, greater thickness, smaller base, and is located on the nasal side of the eye. Tumours located near the optic disc and foveola, those with lesser thickness, larger base, and located on the temporal side are likely to be associated with considerable visual impairment following local resection and, in such cases, other methods such as radiotherapy or enucleation should be considered. Younger individuals tend to tolerate better the hypotensive anaesthesia and have less vascular disease that might contribute to intraoperative or postoperative bleeding. Furthermore, the gelatinous vitreous in younger patients provides better retinal tamponade. Tumours with greater basal diameter and less thickness are best treated with radioactive plaques, perhaps combined with laser or thermotherapy.

When the patient is older, has systemic vascular disease, is taking systemic anticoagulants or when the tumour has a larger base, less thickness, and is more posteriorly located, it may be more advisable to recommend plaque radiotherapy or enucleation. Local resection in such cases is more difficult and more likely to result in intraoperative and postoperative complications such as vitreous haemorrhage, subretinal haemorrhage, retinal detachment, and severe visual impairment. In addition, the reports of Damato and associates suggest that local recurrence and systemic metastasis may be greater in such cases.

More specifically, we believe that local resection can be favoured when the patient is less than 65 years of age and in good health; the tumour thickness is greater than $8 \mathrm{~mm}$, its diameter is less than $15 \mathrm{~mm}$, its posterior margin is more than $4 \mathrm{~mm}$ from the foveola and $3 \mathrm{~mm}$ from the optic disc, and has a nasal location. We believe that local resection is relatively contraindicated for patients older than 65 years, when the tumour is less than $8 \mathrm{~mm}$ in thickness, more than $15 \mathrm{~mm}$ in diameter, less than $4 \mathrm{~mm}$ from the foveola and $3 \mathrm{~mm}$ from the optic disc, and has a temporal location. Most such tumours are best treated with plaque radiotherapy, thermotherapy, or a combination of those two methods.

It is quite certain that most posterior uveal melanomas will continue to be managed by radiotherapy or enucleation and that local resection will be reserved for cases that meet the relatively strict criteria outlined above. However, we believe currently that local resection has a definite place in the management of selected posterior uveal melanomas and that there are frequent instances where it is clearly the treatment of choice. However, the surgery is very difficult and there are numerous potential complications. Therefore, this procedure should only be performed at institutions where there are experienced surgeons who have the frequent opportunities to perform such surgery. It is quite likely that more patients undergoing local resection for uveal melanoma will receive supplemental treatment with radiotherapy, photocoagulation, thermotherapy, or other adjunctive measures in the future. Foulds and Damato have pioneered the development and perfection of local resection of uveal melanoma and they are to be congratulated for their continued contributions.

Supported by the Eye Tumor Research Foundation, Philadelphia, PA, USA.

Ocular Oncology Service,

JERRY A SHIELDS

Wills Eye Hospital,

Thomas Jefferson University,

Philadelphia, USA

Zimmerman LE, McLean IW, Foster WD. Does enucleation of the eye containing a malignant melanoma prevent or accelerate the dissemination of taining a malignant melanoma prevent or accelera

2 Shields JA, Shields CL. Intraocular tumours. A text and atlas. Philadelphia: WB Saunders, 1992: 171-205.

3 Shields JA, Shields CL. Surgical approach to lamellar sclerouvectomy for posterior uveal melanomas. The 1986 Schoenberg lecture. Ophthalmic Surg 1988; 19: 774-80.

4 Shields JA, Shields CL, Shah P, Sivalingam V. Partial lamellar sclerouvectomy for ciliary body and choroidal tumours. Ophthalmology 1991; 98 971-83.

5 Foulds WS, Damato BE. Low-energy long-exposure laser therapy in the management of choroidal melanoma. Graefes Arch Clin Exp Ophthalmol 1986; 224: 26-31.

6 Oosterhuis JA, Journee-de Korver H, Kakebeeke-Keeme HM, Bleeker JC. Transpupillary thermotherapy in choroidal melanoma. Arch Ophthalmol 1995; 113: 315-21. 